\title{
Combining Ability Studies on Yield and Quality Related Traits and Reaction to Bacterial Wilt Resistance in Tomato
}

\author{
V. Harshavardhan Gowda* and P.R. Dharmatti \\ Department of Horticulture, University of Agricultural Sciences, Dharwad, Karnataka, India \\ *Corresponding author
}

\section{A B S T R A C T}

The experiment was conducted in Department of Horticulture, Agriculture College, Dharwad during the year 2017-18. Fifty crosses were evolved by crossing 10 females

\section{Keywords}

Combining ability, Line $\mathrm{x}$

Tester analysis, General

combining ability,

Specific combing ability

Article Info

Accepted:

12 July 2018

Available Online:

10 August 2018 (lines) with five males (Testers) in line $\mathrm{x}$ tester fashion and were evaluated along with the parents and commercial check in randomized block design. Data was been subjected to line $\mathrm{x}$ tester analysis. Analyses of variance were found significant for crosses with respect to yield and quality parameters studied. All the parameters under investigation have recorded significant gca and sca effects in both direction indicating presence of both additive and non-additive components. Among the lines and tester evaluated DMT-3 and PRDS were identified has best general combiners respectively indicating additivity for the parameters. Yield per plant has recorded significant high sca effect in DMT-4 x PRDS which is also found significant for fruits per plant and average fruit weight. Overall significant sca effect was recorded in L-27 x Arka Saurabh for maximum parameters. Fifty crosses evolved by parental combination of resistant $\mathrm{x}$ resistant and resistant $\mathrm{x}$ susceptible for bacterial wilt showed high resistant to the disease indicating the dominant gene governing the resistance.

\section{Introduction}

Tomato (Solanum lycopersicum L.) $(2 \mathrm{n}=2 \mathrm{x}=$ $24)$ is an important and widely grown solanaceous vegetable crop around the world. India ranks second in the world next to China with annual production of 18735.9 thousand $\mathrm{mt}$ and productivity of $21.2 \mathrm{t} / \mathrm{ha}$. In the tropics, tomato productivity is comparatively low due to attack of several diseases and pest. Among Major bacterial diseases of tomato and other solanaceous crops, bacterial wilt is considered as the most serious. Bacterial wilt of tomato caused Ralstonia solanacearum has provided many enigmas for scientists working on tomato and other crop species. Even though several management strategies are proposed, development of genetically resistant hybrids with good horticultural traits is the most economical and eco-friendly approach to mitigate the yield loss due bacterial wilt.

The concept of combing ability is the landmark in the hybridization programme. Combining ability analysis is one of the effective approaches available for estimating the combining ability effects that helps in selecting desirable parents and crosses for the 
exploitation of heterosis. In order to identify potential cross combinations, it is very important to screen out the parent materials for combining ability. With this background study of combining ability for yield and quality related traits and reaction to bacterial wilt in Tomato was taken.

\section{Materials and Methods}

The experimental material for this study comprised of 15 genotypes which were selected based on their Per se performance for yield and reaction to bacterial wilt disease.

From these 15 genotypes, 50 crosses were evolved in a line $\mathrm{x}$ tester design with ten genotypes as female (lines)viz., DMT-3, DMT-4, L-16,L-27, L-33, L-36, L-37, L-9, L24 and Line-10 which exhibited resistance to bacterial wilt disease with desirable horticultural traits and five genotypes as male (testers) viz., Arka Saurabh, Arka Abha, Arka Alok, HDT-1 and PRDS, all are resistant to bacterial wilt disease except HDT-1 which has shown susceptible reaction. The Evaluation of 50 hybrids, 15 parents, along with commercial check NS 2535 was carried out during 2017 July and observation was recorded. Combing ability analysis was carried out according to the formulae given by Kempthorne (1957) through WINDOWSTAT (edition 9.1).

\section{Results and Discussion}

In the present investigation all the 50 hybrids were found completely free from wilt incidence because the parents (Males and Females) used were free from bacterial wilt except HDT-1. The resistant reaction in the hybrids was due to the dominance of the trait since these are $\mathrm{R} \times \mathrm{R}$ and $\mathrm{R} \times \mathrm{S}$ combinations for bacterial wilt. Sharma and Akshay, (2011), Prasana, (2012) and Smitha, (2015), and have found similar results.
The analysis of variance of combing ability revealed that the presence of both additive and non-additive gene action in all characters. The variance due to $s c a$ was higher in magnitude than $g c a$ for all the traits except for days to first and fifty per cent flowering and number of fruits per plant. Further values for ratio of gca to $s c a$ variance for all the traits support predominance of non-additive gene effects governing the expressions of most of the characters (Table 1).

In the present study, magnitude of sca was greater than magnitude of $\mathrm{gca}$ and was highest for plant height, average fruit weight, number of branches, total soluble solids, number of locules per fruit, pericarp thickness, and yield per plant indicating more non additive gene action for yield and quality attributes, whereas magnitude of sca and was highest for number of fruits per plant, days to first flowering and days to fifty per cent flowering. The ratio of gcalsca was less then unity for all the crosses except plant height, number of fruits per plant, days to first and fifty percent flowering indicating the preponderance of dominance, additive $\times$ additive, dominance $\times$ dominance type of gene action (Table 2). Total variance due to crosses can be partitioned into variance due to lines, testers and their interaction effects. The contribution of lines compared to testers was more for the only trait plant height, whereas, testers contributed to a greater extent with days to first flowering, days to fifty percent flowering and number of fruits per plant. The female and male interaction was higher than their parents for the traits like number of branches, average fruit weight, yield per plant, total soluble solids, pericarp thickness and number of locules indicating that interaction of genes in hybrids played a major role in the expression of these traits. The results of the study were supported by the earlier findings of Virupannavar (2009), Singh and Asati (2011), Akshay (2011), Kumar et al., (2016) and Salve et al., (2017). 
Table.1 Analysis of variance in respect to 10 characters for combining ability studies

\begin{tabular}{|c|c|c|c|c|c|c|c|}
\hline Parameters & Replicates & Crosses & Line Effect & Tester Effect & Line $\times$ Tester Effect & Error & Total \\
\hline DF & 1 & 49 & 9 & 4 & 36 & 49 & 99 \\
\hline PH & 0.918 & $298.766 * *$ & $696.319 * *$ & $1030.265^{* *}$ & $118.101 * *$ & 3.219 & 149.77 \\
\hline NOB & $2.986 *$ & $2.217 * *$ & 3.118 & $5.643 *$ & $1.611 * *$ & 0.089 & 1.172 \\
\hline DFF & 1 & $3.940 * *$ & 0.76 & $39.085 * *$ & $0.829 *$ & 0.449 & 2.182 \\
\hline $\mathrm{DF} \% \mathrm{~F}$ & 0.490 & $2.976 * *$ & 0.29 & $31.715 * *$ & 0.454 & 0.429 & 1.690 \\
\hline NFPP & $115.348 *$ & $95.648 * *$ & $121.896 * *$ & $563.335 * *$ & $37.121 * *$ & 3.008 & 49.995 \\
\hline $\mathbf{A W F}$ & 0.154 & $130.291 * *$ & 174.342 & 264.791 & $104.334 * *$ & 1.768 & 65.358 \\
\hline YPP & $0.007 *$ & $0.426 * *$ & 0.295 & $2.333 * *$ & $0.247 * *$ & 0.001 & 0.212 \\
\hline TSS & 0.005 & $1.524 * *$ & 1.162 & $3.682 *$ & $1.375 * *$ & 0.029 & 0.769 \\
\hline PCT & 1.228 & $1.401 * *$ & 2.235 & 2.733 & $1.044 * *$ & 0.05 & 0.731 \\
\hline NOL & 0 & $1.550 * *$ & 1.47 & $5.27 * *$ & $1.150 * *$ & 0.00 & 0.767 \\
\hline
\end{tabular}

*Significant at $5 \% \quad * *$ Significant at $1 \%$

\begin{tabular}{|c|c|c|c|c|}
\hline PH- Plant height & DFF- Days to first flowering & $\begin{array}{l}\text { NFPP- number of fruits per } \\
\text { plant }\end{array}$ & YPP-Yield per plant & PC- Pericarp thickness \\
\hline $\begin{array}{l}\text { NOB- Number of } \\
\text { branches }\end{array}$ & DF\%F - Days to $50 \%$ flowering & AFW-Average fruit weight & $\begin{array}{l}\text { TSS- Total soluble } \\
\text { solids }\end{array}$ & NOL- number of locules \\
\hline
\end{tabular}


Table. 2 Contribution of lines, testers and line $\times$ tester and estimates of variance components for different quantitative characters in tomato

\begin{tabular}{|c|c|c|c|c|c|c|}
\hline \multirow[t]{2}{*}{ Characters } & \multicolumn{3}{|c|}{ Per cent contribution } & \multicolumn{2}{|c|}{ Variance } & \multirow{2}{*}{$\begin{array}{c}\text { Ratio } \\
\text { GCA/SCA }\end{array}$} \\
\hline & Line & Tester & Line $\times$ Tester & GCA & SCA & \\
\hline DF & 9 & 4 & 36 & & & \\
\hline PH & 42.80 & 28.1502 & 29.042 & 57.358 & 57.587 & 1 \\
\hline NOB & 25.846 & 20.755 & 53.397 & 0.285 & 0.755 & 0.38 \\
\hline DFF & 3.5433 & 80.98 & 15.46 & 1.305 & 0.238 & 5.48 \\
\hline $\mathrm{DF} \% \mathrm{~F}$ & 1.79 & 87.00 & 11.20 & 1.044 & 0.059 & 17.69 \\
\hline NFPP & 23.40 & 48.07 & 28.51 & 22.647 & 17.108 & 1.32 \\
\hline AWF & 24.57 & 16.59 & 58.80 & 14.532 & 51.378 & 0.28 \\
\hline YPP & 12.705 & 44.69 & 44.60 & 0.088 & 0.123 & 0.72 \\
\hline TSS & 14.00 & 19.71 & 66.27 & 0.160 & 0.675 & 0.24 \\
\hline PCT & 29.30 & 15.92 & 54.76 & 0.163 & 0.501 & 0.33 \\
\hline NOL & 17.49 & 27.76 & 54.74 & 0.224 & 0.568 & 0.39 \\
\hline * Significant at 5 & $* *$ Signi & & & & & \\
\hline lant height & DFF- D & rst flowering & $\begin{array}{l}\text { NFPP-number of fruit } \\
\text { plant }\end{array}$ & YPP- & per plant & PC- Pericarp thickness \\
\hline $\begin{array}{l}\text { Number of } \\
\text { hes }\end{array}$ & $\mathrm{DF} \% \mathrm{~F}-$ & $50 \%$ flowering & AFW-Average fruit wei & $\begin{array}{l}\text { TSS- } \\
\text { solids }\end{array}$ & soluble & NOL- number of locules \\
\hline
\end{tabular}


Table.3 Estimation of general combing ability effect for ten characters (Line $\times$ Tester) in tomato genotypes

\begin{tabular}{|c|c|c|c|c|c|c|c|c|c|c|c|}
\hline Parameters & \multirow[t]{2}{*}{ PH } & \multirow[t]{2}{*}{ NOB } & \multirow[t]{2}{*}{ DFF } & \multirow[t]{2}{*}{$\mathrm{DF} \% \mathrm{~F}$} & \multirow[t]{2}{*}{ NFPP } & \multirow{2}{*}{\multicolumn{2}{|c|}{ AFW }} & \multirow[t]{2}{*}{ YPP } & \multirow[t]{2}{*}{ TSS } & \multirow[t]{2}{*}{ PCT } & \multirow[t]{2}{*}{ NOL } \\
\hline Parents & & & & & & & & & & & \\
\hline \multicolumn{12}{|c|}{ Lines } \\
\hline DMT-3 & $3.338 * *$ & $0.680 * *$ & 0.04 & -0.23 & $2.487 * *$ & \multicolumn{2}{|c|}{$1.573 * *$} & $0.236 * *$ & $0.493 * *$ & $0.854 * *$ & $-0.782 * *$ \\
\hline DMT-4 & -0.03 & $-0.234 *$ & $0.540 * *$ & 0.07 & 0.881 & \multicolumn{2}{|c|}{$3.257 * *$} & $0.244^{* *}$ & $-0.289 * *$ & $-0.635 * *$ & $0.198 * *$ \\
\hline L-16 & $-8.078 * *$ & $-0.495 * *$ & -0.06 & 0.07 & 0.167 & \multicolumn{2}{|c|}{$-5.233 * *$} & $-0.071 * *$ & $-0.473 * *$ & $0.468 * *$ & $0.198 * *$ \\
\hline L-27 & $-5.322 * *$ & $0.612 * *$ & 0.14 & 0.27 & $-3.613 * *$ & \multicolumn{2}{|c|}{-0.109} & $-0.155^{* *}$ & $0.381 * *$ & $-0.583 * *$ & $0.398 * *$ \\
\hline L-33 & $-1.926 * *$ & -0.027 & 0.34 & 0.07 & -0.359 & \multicolumn{2}{|c|}{$-6.613 * *$} & $-0.167 * *$ & $0.293 * *$ & $-0.231 * *$ & -0.002 \\
\hline L-36 & $-4.880 * *$ & $0.784 * *$ & -0.06 & 0.07 & $2.847 * *$ & \multicolumn{2}{|c|}{$7.329 * *$} & $0.103^{* *}$ & $0.191 * *$ & $-0.159 *$ & $0.198 * *$ \\
\hline L-37 & $4.328 * *$ & $-0.627 * *$ & -0.36 & -0.23 & $-6.333^{* *} *$ & \multicolumn{2}{|c|}{0.389} & $-0.210^{* *}$ & $-0.279 * *$ & $0.366^{* *}$ & $0.198 * *$ \\
\hline L-9 & $13.332 * *$ & $-0.233 *$ & -0.16 & -0.13 & $-2.833^{* *}$ & -3.713 & & $-0.159 * *$ & $-0.375^{* *}$ & $-0.269 * *$ & -0.002 \\
\hline L-10 & $-12.580 * *$ & $-0.731 * *$ & -0.26 & -0.13 & $5.667 * *$ & 1.249 & & $0.059 * *$ & 0.093 & -0.009 & $0.198 * *$ \\
\hline L-24 & $11.820 * *$ & $0.269 *$ & -0.16 & 0.17 & $1.087 *$ & $1.867^{*}$ & & $0.138^{* *}$ & -0.033 & $0.198 * *$ & $-0.602 * *$ \\
\hline & & & & & ters & & & & & & \\
\hline Arka Saurabh & $8.122 * *$ & $0.253^{* *}$ & $2.190 * *$ & $1.870^{* *}$ & $-3.163^{* *}$ & 0.504 & & $-0.090 * *$ & $-0.169 * *$ & $-0.494 * *$ & $-0.292 * *$ \\
\hline ArkaAbha & $-5.314 * *$ & $-0.246 * *$ & $-0.710 * *$ & $-0.980 * *$ & $-5.236^{* *}$ & -2.199 & & $-0.345 * *$ & $0.255^{* *}$ & $0.234 * *$ & $0.398 * *$ \\
\hline HDT-1 & $-5.378 * *$ & $-0.601 * *$ & -0.16 & $-0.330 *$ & $-0.823 *$ & -4.519 & & $-0.175^{* *}$ & $-0.282 * *$ & $-0.135 * *$ & $-0.402 * *$ \\
\hline ArkaAlok & $-5.024 * *$ & $-0.185 *$ & $-1.560 * *$ & $-1.180^{* *}$ & 0.607 & $1.087 *$ & & $0.56^{* *}$ & $-0.423 * *$ & -0.078 & $0.698 * *$ \\
\hline PRDS & $7.595^{* *}$ & $0.780 * *$ & 0.24 & $0.620 * *$ & $8.614 * *$ & $5.125^{*}$ & & $0.533^{* *}$ & $0.620 * *$ & $0.474 * *$ & $-0.402 * *$ \\
\hline CD 5\% GCA (L) & 1.087 & 0.204 & 0.377 & 0.368 & 1.083 & 0.796 & & 0.020 & 0.099 & 0.131 & 0.086 \\
\hline CD $5 \%$ GCA (Tester) & 0.769 & 0.144 & 0.267 & 0.26 & 0.766 & 0.563 & & 0.014 & 0.07 & 0.093 & 0.061 \\
\hline$*$ Significant at & ** Significan & $t 1 \%$ & & & & & & & & & \\
\hline PH- Plant height & DFF- & ays to first & owering & $\begin{array}{l}\text { NFPP- nt } \\
\text { plant }\end{array}$ & lber of frt & ts per & & P-Yield per & & Pericarp $t$ & ckness \\
\hline $\begin{array}{l}\text { NOB- Number of } \\
\text { branches }\end{array}$ & $\mathrm{DF} \% \mathrm{I}$ & Days to 50 & flowering & $A F W-A v$ & rage fruit $\mathrm{n}$ & ight & & $\begin{array}{l}\text { - Total solı } \\
\text { ds }\end{array}$ & & - number 0 & ocules \\
\hline
\end{tabular}


Table.4 Estimation of specific combing ability effects for ten characters in (Line $\times$ Tester) in tomato hybrids

\begin{tabular}{|c|c|c|c|c|c|c|c|c|c|c|}
\hline & PH & NOB & DFF & DF\%F & NFPP & AWF & YPP & TSS & PCT & NOL \\
\hline DMT-3 × Arka Saurabh & 1.932 & $-0.708 * *$ & $1.410 * *$ & 0.73 & -1.477 & $5.210 * *$ & $0.082 * *$ & -0.211 & $0.413 * *$ & $0.972 * *$ \\
\hline DMT-3 $\times$ ArkaAbha & $-2.782 *$ & -0.009 & $-1.190 * *$ & $-0.920 *$ & 1.796 & $-12.387 * *$ & $-0.263 * *$ & $1.865 * *$ & 0.037 & 0.182 \\
\hline DMT-3 x HDT-1 & $4.382 * *$ & $-0.704 * *$ & -0.24 & -0.07 & -1.017 & $7.933 * *$ & $0.147 * *$ & $-0.598 * *$ & $-0.645 * *$ & -0.018 \\
\hline DMT-3 × ArkaAlok & $-2.992 *$ & $2.055 * *$ & -0.34 & 0.28 & $-2.947 *$ & $-6.543 * *$ & $-0.299 * *$ & $-1.157 * *$ & -0.001 & $-1.118 * *$ \\
\hline DMT-3 $\times$ PRDS & -0.541 & $-0.635 * *$ & 0.36 & -0.02 & $3.646 * *$ & $5.789 * *$ & $0.334 * *$ & 0.1 & 0.196 & -0.018 \\
\hline DMT-4 × Arka Saurabh & 2.4 & 0.106 & -0.09 & -0.57 & $2.429 *$ & $2.786 * *$ & $0.229 * *$ & -0.039 & -0.009 & -0.108 \\
\hline DMT-4 $\times$ ArkaAbha & 1.736 & $-0.995 * *$ & 0.81 & $1.280 * *$ & -1.498 & $-2.731 * *$ & $-0.181 * *$ & $0.247 *$ & $0.384 *$ & $0.202 *$ \\
\hline DMT-4 × HDT-1 & $-6.900 * *$ & $1.160 * *$ & -0.74 & -0.37 & $-3.911 * *$ & $-6.011 * *$ & $-0.356 * *$ & $-0.816 * *$ & $-1.857 * *$ & 0.002 \\
\hline DMT-4 × ArkaAlok & 1.136 & -0.396 & 0.16 & -0.02 & $-4.341 * *$ & $-8.647 * *$ & $-0.392 * *$ & $0.525 * *$ & $0.747 * *$ & -0.098 \\
\hline DMT-4 $\times$ PRDS & 1.627 & 0.124 & -0.14 & -0.32 & $7.322 * *$ & $14.605 * *$ & $0.701 * *$ & 0.082 & $0.735 * *$ & 0.002 \\
\hline L-16 × Arka Saurabh & $-2.852 *$ & -0.233 & $-0.990 *$ & -0.07 & $-2.957 *$ & $-3.184 * *$ & $-0.191 * *$ & $1.075 * *$ & 0.02 & -0.108 \\
\hline L-16 x ArkaAbha & 0.584 & $0.566 *$ & -0.09 & -0.22 & -0.084 & $3.619 * *$ & $0.209 * *$ & $-1.569 * *$ & $-0.498 * *$ & $0.202 *$ \\
\hline L-16 $\times$ HDT-1 & -2.352 & -0.079 & 0.36 & 0.13 & $-2.597 *$ & $3.239 * *$ & $-0.121 * *$ & $-0.882 * *$ & $0.341 *$ & 0.002 \\
\hline L-16 x ArkaAlok & $-3.056 *$ & $-0.595 *$ & -0.24 & -0.02 & $6.273 * *$ & $7.133 * *$ & $0.508 * *$ & $0.509 * *$ & -0.266 & -0.098 \\
\hline L-16 $\times$ PRDS & $7.675 * *$ & 0.34 & $0.960 *$ & 0.18 & -0.634 & $-10.805 * *$ & $-0.404 * *$ & $0.866 * *$ & $0.403 * *$ & 0.002 \\
\hline L-27 × Arka Saurabh & $-12.708 * *$ & $0.750 * *$ & -0.19 & -0.27 & $5.923 * *$ & $3.692 * *$ & $0.448 * *$ & $0.701 * *$ & $0.809 * *$ & $0.692 * *$ \\
\hline L-27 x ArkaAbha & -0.022 & $1.389 * *$ & 0.71 & 0.58 & $4.596 * *$ & $9.395 * *$ & 0.323 & -0.163 & -0.248 & 0.002 \\
\hline L-27 x HDT-1 & $6.892 * *$ & 0.069 & 0.16 & -0.07 & -1.917 & $-4.285 * *$ & $-0.167 * *$ & $-0.786 * *$ & -0.279 & $-1.198 * *$ \\
\hline L-27 × ArkaAlok & $5.918 * *$ & $-1.622 * *$ & -0.44 & -0.22 & $2.753 *$ & $-3.651 * *$ & 0.002 & $0.635 * *$ & $0.335 *$ & $-0.298 * *$ \\
\hline L-27 x PRDS & -0.081 & $-0.587 *$ & -0.24 & -0.02 & $-11.354 * *$ & $-5.149 * *$ & $-0.605^{* *}$ & $-0.388 * *$ & $-0.618 * *$ & $0.802 * *$ \\
\hline L- $33 \times$ Arka Saurabh & $3.996 * *$ & -0.361 & -0.39 & -0.07 & $-3.631 * *$ & $-4.104 * *$ & $-0.250 * *$ & $-0.451 * *$ & $0.748 * *$ & 0.092 \\
\hline L-33 $\times$ ArkaAbha & -0.968 & 0.398 & -0.49 & -0.22 & -0.088 & $-4.601 * *$ & -0.025 & $-0.235 *$ & $0.560 * *$ & $0.402 * *$ \\
\hline L-33 $\times$ HDT-1 & -0.364 & $1.053 * *$ & -0.04 & 0.13 & $4.829 * *$ & 1.319 & $0.345 * *$ & $1.002 * *$ & 0.119 & $0.202 *$ \\
\hline L-33 x ArkaAlok & $-3.688 * *$ & -0.163 & $1.360 * *$ & -0.02 & -0.001 & 1.713 & $0.164 * *$ & $-0.377 * *$ & $-1.258 * *$ & 0.102 \\
\hline L-33 $\times$ PRDS & 1.023 & $-0.928 * *$ & -0.44 & 0.18 & -1.108 & $5.675 * *$ & $-0.233 * *$ & 0.06 & -0.169 & $-0.798 * *$ \\
\hline L-36 × Arka Saurabh & $-7.050 * *$ & $0.788 * *$ & 0.01 & -0.07 & 0.263 & -0.746 & $-0.125^{* *}$ & -0.209 & -0.124 & $-1.108 * *$ \\
\hline L-36 × ArkaAbha & $5.486 * *$ & -0.338 & -0.09 & -0.22 & $-2.864 *$ & $-3.643 * *$ & $-0.190 * *$ & $-0.513 * *$ & 0.189 & $0.202 *$ \\
\hline
\end{tabular}




\begin{tabular}{|c|c|c|c|c|c|c|c|c|c|c|}
\hline & & & & & & & & & & ntinued... \\
\hline & $\mathbf{P H}$ & NOB & DFF & DF\%F & NFPP & AWF & YPP & TSS & PCT & NOL \\
\hline L-36 $\times$ HDT-1 & $3.540 * *$ & -0.228 & 0.36 & 0.13 & -2.277 & $-6.723 * *$ & $-0.255^{* *}$ & $0.504 * *$ & $0.767 * *$ & 0.002 \\
\hline L-36 × ArkaAlok & $-4.954 * *$ & -0.184 & -0.24 & -0.02 & $-2.607 *$ & $10.081 * *$ & 0.029 & $0.445 * *$ & $-0.690 * *$ & $1.902 * *$ \\
\hline L-36 × PRDS & $2.977 *$ & -0.039 & -0.04 & 0.18 & $7.486 * *$ & 1.033 & $0.542 * *$ & $-0.228 *$ & -0.142 & $-0.998 * *$ \\
\hline L- $37 \times$ Arka Saurabh & $18.342 * *$ & $-0.481 *$ & 0.31 & 0.23 & 2.343 & $4.394 * *$ & $0.198 * *$ & $-0.979 *:$ & $0.691 * *$ & -0.108 \\
\hline L-37 × ArkaAbha & -1.422 & -0.002 & 0.21 & 0.08 & 1.316 & $2.897 * *$ & $0.203 * *$ & $1.337 * *$ & $-1.306 * *$ & $0.202 *$ \\
\hline L-37 x HDT-1 & -2.208 & 0.453 & 0.66 & 0.43 & -1.697 & 0.217 & $-0.122 * *$ & $0.474 * *$ & -0.017 & 0.002 \\
\hline L-37 × ArkaAlok & $-7.282 * *$ & 0.437 & 0.06 & 0.28 & $3.473 * *$ & $6.821 * *$ & $0.362 * *$ & $0.405 * *$ & $0.497 * *$ & -0.098 \\
\hline L-37 x PRDS & $-7.431 * *$ & -0.408 & $-1.240 * *$ & $-1.020 *$ & $-5.434 * *$ & $-14.327 * *$ & $-0.640 * *$ & $-1.238 *:$ & 0.135 & 0.002 \\
\hline L- $9 \times$ Arka Saurabh & 1.238 & 0.155 & 0.11 & 0.13 & -2.357 & $6.296 * *$ & $0.112 * *$ & $0.287 *$ & $-1.055 * *$ & 0.092 \\
\hline L-9 $\times$ ArkaAbha & $4.674 * *$ & 0.004 & 0.01 & -0.02 & -1.784 & $1.999 *$ & 0.042 & -0.027 & $0.849 * *$ & $0.402 * *$ \\
\hline L-9 $\times$ HDT-1 & $-7.262 * *$ & $-0.941 * *$ & 0.46 & 0.33 & 0.403 & $-3.681 * *$ & -0.033 & $0.700 * *$ & $0.377 *$ & $-0.798 * *$ \\
\hline L-9 × ArkaAlok & $14.484 * *$ & $0.943 * *$ & -0.14 & 0.18 & $4.873 * *$ & -1.187 & $0.0666 * *$ & $-1.629 *:$ & 0.021 & $-0.898 * *$ \\
\hline L-9 $\times$ PRDS & $-13.135 * *$ & -0.162 & -0.44 & -0.62 & -1.134 & $-3.425 * *$ & $-0.186^{* *}$ & $0.668 * *$ & -0.192 & $1.202 * *$ \\
\hline L- $10 \times$ Arka Saurabh & $-12.750 * *$ & -0.017 & 0.21 & 0.13 & 1.143 & $-8.256 * *$ & -0.226 & -0.011 & -0.164 & -0.108 \\
\hline L-10 × ArkaAbha & $-8.174 * *$ & -0.398 & 0.11 & -0.02 & 1.416 & $4.037 * *$ & 0.014 & $-0.335 *$ & $-0.351 *$ & $-1.798 * *$ \\
\hline L-10 $\times$ HDT-1 & 0.32 & $-1.243 * *$ & -0.44 & -0.17 & 1.903 & -0.843 & 0.009 & 0.102 & 0.163 & $1.002 * *$ \\
\hline L-10 x ArkaAlok & $11.126 * *$ & $-0.759 * *$ & -0.04 & -0.32 & $-5.527 * *$ & 0.951 & $-0.187 * *$ & $0.343 * *$ & $0.302 *$ & $0.902 * *$ \\
\hline L-10 $\times$ PRDS & $9.477 * *$ & $2.416 * *$ & 0.16 & 0.38 & 1.066 & $4.113 * *$ & $0.391 * *$ & -0.1 & 0.05 & 0.002 \\
\hline L- $24 \times$ Arka Saurabh & $7.450 * *$ & 0.003 & -0.39 & -0.17 & -1.677 & $-6.084 * *$ & $-0.275^{* *}$ & -0.165 & $-1.331 * *$ & $-0.308 * *$ \\
\hline L-24 × ArkaAbha & 0.886 & $-0.618 * *$ & 0.01 & -0.32 & $-2.804 *$ & 1.419 & $-0.130 * *$ & $-0.609 *$ & $0.383 *$ & 0.002 \\
\hline L-24 x HDT-1 & $3.950 * *$ & $0.457 *$ & -0.54 & -0.47 & $6.283 * *$ & $8.839 * *$ & $0.555^{* *}$ & $0.298 * *$ & $1.031 * *$ & $0.802 * *$ \\
\hline L-24 × ArkaAlok & $-10.694 * *$ & 0.281 & -0.14 & -0.12 & -1.947 & $-6.667 * *$ & $-0.251 * *$ & $0.299 * *$ & $0.314 *$ & $-0.298 * *$ \\
\hline L-24 x PRDS & -1.593 & -0.124 & $1.060 *$ & $1.080 *$ & 0.146 & $2.495 * *$ & $0.102 * *$ & 0.176 & $-0.397 * *$ & $-0.198 *$ \\
\hline CD At 5\% & 2.431 & 0.455 & 0.844 & 844 & 2.422 & 1.78 & 0.046 & 0.22 & 0.294 & 0.192 \\
\hline$*$ Significar & ** Significant a & & & & & & & & & \\
\hline PH- Plant height & DFF- Days & to first flower & & PP- number & f fruits per $p$ & & Yield per ple & & Pericarp thi & ness \\
\hline NOB- Number of branches & DF\%F - Day & s to $50 \%$ flow & ering & W- Average & uit weight & TSS & Total soluble & & - number of 1 & cules \\
\hline
\end{tabular}


An overview of the estimates of gca of parents (Table 3) revealed that the genotype DMT-3 and PRDS exhibited desirable significant gca for 8 parameters yield per plant, average fruit weight, number of fruits, number of branches, plant height and quality parameters viz., total soluble solids, pericarp thickness and number of locules per fruit whereas, L-24 also recorded significant positive gca effect for above parameters except total soluble solids. Crosses involving this parent may produce good hybrids with better quality since it was identified as a good general combiner for the most of the traits. The results of the present study for this trait were supported by earlier findings of Kulkarni (2003), Virupannavar (2009), Akshay (2011), Reddy et al., (2017), Salve et al., (2017) and Gautam et al., (2018).

The combing ability analysis for yield and yield related parameters revealed that, both additive and non-additive effects as important in the expression of the traits with the predominance of non-additivity as evidenced by combing ability variance and estimates. This was further confirmed by significant sca effects of the cross studied.

Overall specific combing ability estimates of crosses (Table 4) indicated significant positive sca effects for yield related parameters in DMT-3 $\times$ PRDS, DMT- $4 \times$ PRDS, DMT-4 $\times$ Arka Saurabh, L-16 $\times$ Arka Alok, L-27 × Arka Saurabh, L-37 × Arka Alok. Majority of the crosses which showed sca effects in desirable direction for yield had at least one parent with positive gca effect except cross L-27 $\times$ Arka Abha with both parents with negative gca. This elucidates the importance of interaction between the parents and crosses.

Considering all the growth, quality and yield related parameters, Significant sca in desirable direction was recorded with cross L-
$27 \times$ A Saurabh and Line-24 $\times$ HDT-1 with parental combination of low gca $\times$ low gca and high gca $\times$ high gca for over all parameters. Out of 50 crosses indicating predominance of non-additive gene action for these traits (Table 4).

Comprehensive assessment of crosses considering significant sca of 10 crosses in desirable direction revealed that, out of 50 crosses 17 crosses were highly significant sca effects, 13 crosses average significant and 20 crosses low sca effects. Among 17 highly significant crosses seven crosses have parental combination of low gca $\times$ low gca, three crosses with high $\times$ low gca, four crosses with low $\times$ average gca and one cross with low $\times$ high gca. Indicating the high sca resulted due to the line $\times$ tester interaction and per cent contribution of the crosses is more inferring the predominance of additive gene action. Thus the foregoing discussion reveals that in the crosses positive sca effect observed which might be due to complement type of gene effect or due to dominance. Thus genetic interaction might be of additive $\mathrm{x}$ additive non additive, non-additive, non-additive $\mathrm{x}$ non additive and additive $\mathrm{x}$ non additive or gene effect in their heterotic crosses. Additivity is predominant then transgressive breeding is suggested to isolate high yielding lines from the population whereas with non-additive gene action can be exploited through recurrent selection or used as hybrid. Significant sca and gca along with additive and non-additive gene action was reported by Gabrey et al., (2014), Pemba et al., (2014), Reddy et al., (2017) and Salve et al., (2017) for yield related parameters.

Concluding that the top four significant heterotic crosses DMT- $3 \times$ PRDS, DMT $-4 \times$ PRDS, L-36 $\times$ PRDS, L-10 $\times$ PRDS were regarded as superior $F_{1} s$ for yield and yield contributing characters and also free from incidence of bacterial wilt disease and L-27 x 
A Saurabh and Line-24 $\times$ HDT-1 for growth quality and yield. These hybrids after further screening and evaluation can be commercially exploited among tomato growers.

\section{References}

Akshay, A., 2011, Heterosis and combining ability analysis for productivity related traits in tomato. M. Sc. (Agri.) Thesis, Univ. Agric. Sci., Dharwad (India).

Anonymous, 2017. National Horticultural Board, www.indiastat.com.

Gautam, N., Kumar, M., Vikram, A., Kumar, S. and Sharma, S., 2018, Heterosis Studies for Yield and its Components in Tomato (Solanum lycopersicum L.) under North Western Himalayan Region, India Int. J. Curr. Microbiol. App. Sci. 7(2): 1949-1957.

Kempthorne, O., 1957, An Introduction to Genetic Statistics, New York, John Wiley and Sons, 1 st Edn., pp. 456-471.

Kulkarni, G. P., 2003, Investigations on bacterial wilt resistance in tomato.
Ph.D. Thesis, Univ. Agric. Sci., Dharwad.

Kumar, V., and Singh, J. P. and Kaushik, Combining ability studies in tomato (Lycopersicon esculentum L.). Adv. Res. J. Crop Improv., 7(2): 220-223.

Reddy, G. E., Srivastava, K., Reddy, M. P., Reddy, B. R. and Bhandari, H., 2017. Combining ability studies for quality traits in selected tomato (Solanum lycopersicum L.) parents and hybrids. Environ. Ecology. 35(2): 295-300.

Savale, S. V. and Patel, A. I., 2017. Combining ability analysis for fruit yield and quality traits across environments in tomato (Solanum lycopersicum L.) Int. J. Chem. 5(5): 1611-1615.

Virupannavar, H. S., 2009, Genetic studies for productivity and bacterial wilt resistance in tomato. $M . S c$. (Agri.) Thesis, Univ. Agric. Sci., Dharwad (India).

\section{How to cite this article:}

Harshavardhan Gowda, V. and Dharmatti, P.R. 2018. Combining Ability Studies on Yield and Quality Related Traits and Reaction to Bacterial Wilt Resistance in Tomato. Int.J.Curr.Microbiol.App.Sci. 7(08): 1790-1798. doi: https://doi.org/10.20546/ijcmas.2018.708.205 